\title{
Wiederaufnahme des wissenschaftlichen Programms der BGPN
}

Die Corona-Pandemie hat in den letzten Wochen und Monaten unser aller Leben verändert. Aufgrund der Kontaktbeschränkungen war deutschlandweit das gesellschaftliche Leben zum Erliegen gekommen - und damit auch die Veranstaltung von Kongressen, Tagungen und wissenschaftlichen Veranstaltungen. Seit März mussten auch in unserer Gesellschaft die Mittwochsveranstaltungen pausiert werden, die Frühjahrstagung wurde abgesagt.

Wir freuen uns nun, Ihnen mitteilen zu können, dass unter den aktuellen Umständen die Wiederaufnahme unseres Programmes ab September 2020 geplant ist. In den Hörsälen können alle Abstands- und Hygienevorschriften eingehalten werden. Die ausge- fallenen Vorträge werden - ebenso wie die Frühjahrstagung - 2021 nachgeholt.

Bitte beachten Sie die Änderung des gewohnten Veranstaltungsortes der Mittwochsveranstaltungen! Im September und Oktober werden die Veranstaltungen im Kaiserin-Friedrich-Haus am Robert-KochPlatz stattfinden, ab November sind die Veranstaltungen wieder im Hörsaal der Nervenklinik am Campus Charité Mitte geplant. Aktuelle Informationen hierzu erhalten Sie auf unserer Homepage (www.bgpn.de) und über unsern Google-Veranstaltungskalender (bgpnkalender@gmail.com).

Wir freuen uns auf Ihr zahlreiches Erscheinen.

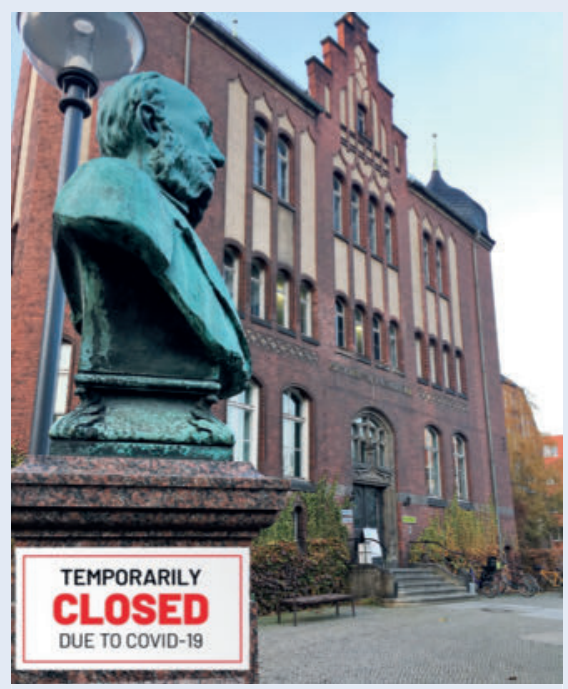

Nervenklinik. Quelle: @Dr. Anja Bauer, Berlin

\section{Wissenschaftliches Programm 2020}

\section{Mittwochsveranstaltungen der BGPN - 18.00h}

\subsubsection{0}

Ort: Kaiserin-Friedrich-Haus, RobertKoch-Platz 7, 10115 Berlin

- Schizophreniebehandlung aus Patientensicht; Dr. Lieselotte Mahler, Kliniken im Theodor-Wenzel-Werk Berlin

- Medikamentenadhärenz in der Epilepsiebehandlung; Prof. Dr. med. Martin Holtkamp, Ev. Krankenhaus Königin Elisabeth Herzberge

18.11.2020

Ort: Hörsaal der Nervenklinik der Charité Campus Mitte

- Neurologische und psychiatrische Falldemonstrationen; Berliner Neurologien und Psychiatrien, in Kooperation mit der Charité - Universitätsmedizin zu Berlin
16.12.2020

Ort: Hörsaal der Nervenklinik der Charité Campus Mitte

- Geschlechtsumwandlungen; Dr. med. Alexander Korte, Klinikum der Universität München

- Religionssensible Psychotherapie; Dr. med. Norbert Mönter, Berlin

\section{Herbsttagung}

Samstag, 31.10.2020 - 10.00 Uhr

Ort: Kaiserin-Friedrich-Haus, RobertKoch-Platz 7, 10115 Berlin

Sprache in der Neurologie und Psychiatrie

- Sprachentwicklungsstörungen; Carolin Schröter, ZAPP Berlin

- Sprache und Schizophrenie: Von der Phänomenologie zur Neurobiologie; Prof. Tilo Kircher, Marburg
- Aphasic Syndromes; Jay P. Mohr, MD, New York

- Sprachzerfall bei Demenz; Prof. Hans Gutzmann, Mainz

- Künstliche Intelligenz und Sprache; Prof. Surjo Soekadar, Berlin

Teilnahme kostenfrei.

\section{IMPRESSUM}

Prof. Dr. Tom Bschor

Redaktion: Dr. Anja M. Bauer

Berliner Gesellschaft für Psychiatrie und Neurologie e. V.

Schlosspark-Klinik, Abteilung für

Psychiatrie

Heubnerweg 2, 14059 Berlin info@bgpn.de,www.bgpn.de 